\title{
Modeling Search Patterns in Species: A Brief Survey of Mathematical Methods
}

\author{
Mauricio Tejo ${ }^{1 *}$ and Sebastian Niklitschek-Soto ${ }^{2}$ \\ ${ }^{1}$ Facultad de Ciencias Naturales y Exactas, Universidad de Playa Ancha \\ ${ }^{2}$ Facultad de Ciencias Físicas y Matemáticas, Universidad de Concepción \\ Email: mauricio.tejo@upla.cl
}

\begin{abstract}
Search patterns in species can give us relevant information about abundance/lack of its food availability. This survey reviews three marked patterns: the Brownian motion, the Lévy flight and an intermediary displacement. After defining the corresponding mathematical models and some of its properties, we consider a general Euler scheme in which these kind of processes can be simulated. Finally, we discuss some possible implications and future researches.
\end{abstract}

Keywords: Markov processes; Brownian motion; Lévy flight; Lévy processes; Heavy-tailed distributions; Euler scheme; Likelihood function.

\section{Introduction}

Movement and dispersal of organisms are of relevant importance in many fields. For some species, it is crucial to recognize its displacement patterns because they give information about the distribution of its food. When food is sparse, the dispersal of such species looks like a Lévy flight (see, e.g., [1], [2], [3], [4], [5]), where the species will need to travel long distances to feed. On the other hand, food abundance creates an impression of a random walk, because the species can feed on either side of its surroundings. In such a case, Brownian motion seems a reasonable model for describing its movement.

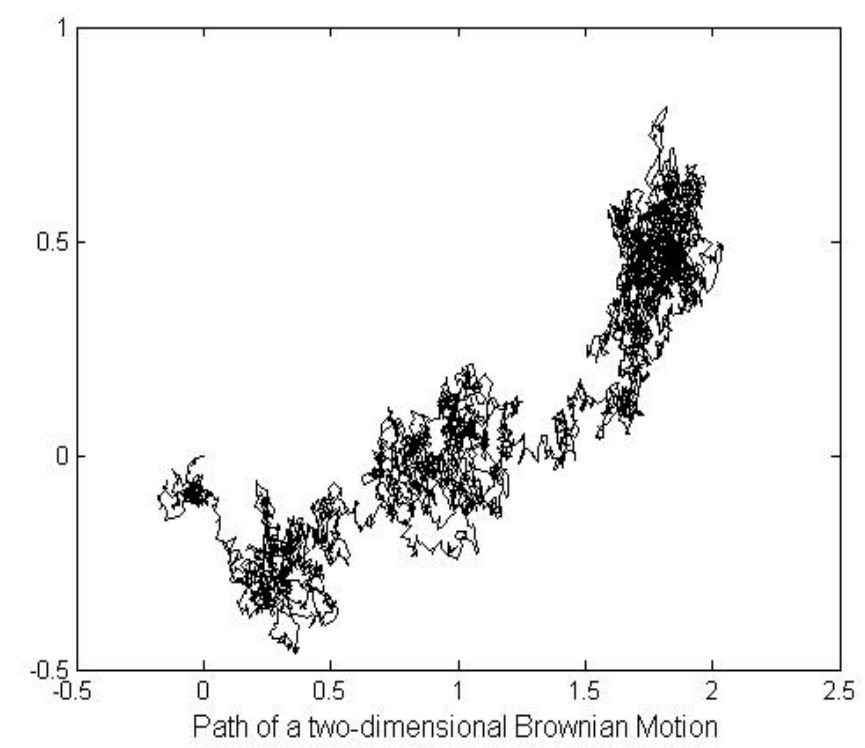

Figure 1. This search pattern pictures abundance of food. 


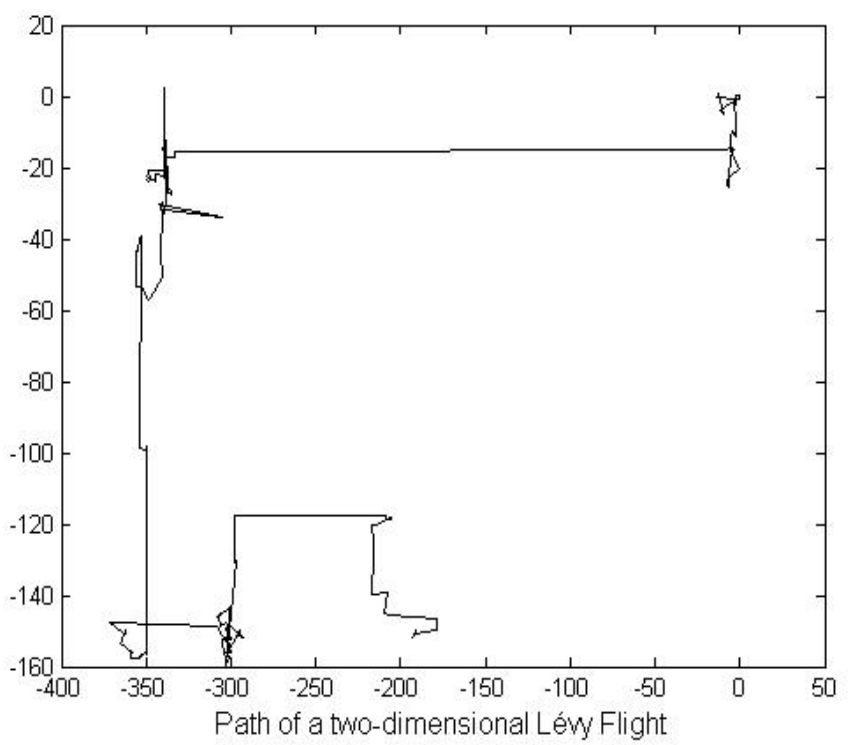

Figure 2. This search pattern pictures lack of food.

Also, an intermediary pattern can be considered when the species have to travel not so long distances for food, probably verifying the beginning of a change in the food distribution. The main aim of this work is to review some mathematical aspects of the modeling of the mentioned displacement patterns and their implications.

The paper is organized as follows: Section 2 focuses on stochastic models that range from the Lévy flight patterns to the Brownian motion pattern. In Section 3, a general Euler scheme for modeling a wide family of stochastic processes is considered. Finally, possible implications and future researches are discussed.

\section{Model Approach}

Roughly, a Lévy flight is a continuous-time jump Markov process whose jumps $X$ follow a heavy-tailed distribution:

$$
\lim _{x \rightarrow \infty} \exp \{\beta x\} P(|X|>x)=\infty
$$

for all $\beta>0$.

A standard real Brownian motion is defined as the continuous-time Markov process $\left\{W_{t}\right\}_{t \in \mathbb{R}_{+}}$with independent increments, characterized by $W_{0}=0$ and for all $t>0, W_{t}$ is distributed as a zero-mean normal random variable with variance equal to $t$ (this is simply denoted as $W_{t} \sim \mathcal{N}(0, t)$ ).

Both Lévy flight and Brownian motion belong to a wider family of stochastic processes: the Lévy processes. These are defined in the following way. Let $\left\{X_{t}\right\}_{t \in \mathbb{R}_{+}}$be a stochastic process defined on a probability space $(\Omega, \mathcal{F}, P)$ adapted to a filtration $\left\{\mathcal{F}_{t}\right\}_{t \in \mathbb{R}_{+}}$, taking values in $\mathbb{R}^{d}$, which has rightcontinuous paths with left-hand limits, usually known as càdlàg processes (French "continue à droite, limite à gauche"; the set of all càdlàg processes on a certain domain $E$ taking values in $M$ is known as the Skorohod space, denoted as $D(E, M))$. We say that $\left\{X_{t}\right\}_{t \in \mathbb{R}_{+}}$is a Lévy process if it satisfies:

1. $P\left(X_{0}=0\right)=1$.

2. For all $t, s \in \mathbb{R}_{+}, X_{t+s}-X_{t} \stackrel{\mathcal{L}}{=} X_{s}$, where "으" means "equals in law or equals in probability distribution".

3. For all $0 \leq s \leq t, X_{t}-X_{s}$ is independent of $\mathcal{F}_{s}$ (i.e., has independent increments). 
Another typical example is the Poisson Process $\left\{N_{t}\right\}_{t \in \mathbb{R}_{+}}$of rate $\lambda>0$, which is a unit pure-jump process satisfying the above requirements. Times between jumps have exponential law of rate $\lambda$.

Lévy processes have a general representation through the characteristic function $(\mathrm{CF})$ of $X_{t}$, for all $t \in \mathbb{R}_{+}$. This representation completely characterizes a Lévy process and is known as the Lévy-Khintchine representation, given by:

$$
\begin{gathered}
X_{t}(u)=E\left(\exp \left\{\mathrm{i} u^{\top} X_{t}\right\}\right) \\
=\exp \left\{t\left[\mathrm{i} u^{\top} \gamma-\frac{1}{2} u^{\top} \Sigma u+\int_{\mathbb{R}^{d} \backslash\{0\}}\left(\exp \left\{\mathrm{i} u^{\top} x\right\}-1-\mathrm{i} u^{\top} x 1_{\{\|x\|<1\}}\right) \nu(d x)\right]\right\},
\end{gathered}
$$

where $u, \gamma \in \mathbb{R}^{d}$, $\top$ is the matrix transpose operator, $\Sigma$ is a $d \times d$ positive semi-definite matrix, $1_{\{\|x\|<1\}}$ is the indicator function on the set $\{\|x\|<1\}$, i.e. $1_{\{\|x\|<1\}}=1$ if $\|x\|<1$ and equal to 0 otherwise, and $\nu$ is a measure (known as Lévy measure), which satisfies $\int_{\mathbb{R}^{d} \backslash\{0\}}(1 \wedge\|x\|) \nu(d x)<\infty$.

Roughly speaking, the previous representation can be decomposed into two notable factors. If for all $t \in \mathbb{R}_{+}$, we write $X_{t}=X_{t}^{\mathrm{c}}+X_{t}^{\mathrm{d}}$, where $\left\{X_{t}^{\mathrm{c}}\right\}_{t \in \mathbb{R}_{+}}$represents the continuous paths of the Lévy process $\left\{X_{t}\right\}_{t \in \mathbb{R}_{+}}$and $\left\{X_{t}^{\mathrm{d}}\right\}_{t \in \mathbb{R}_{+}}$represents its discontinuous paths, we have that $\psi_{X_{t}}(u)=\psi_{X_{t}^{\mathrm{c}}}(u) \psi_{X_{t}^{\mathrm{d}}}(u)$, where $X_{t}^{\mathrm{c}}(u)=\exp \left\{t\left[\mathrm{i} u^{\top} \gamma-\frac{1}{2} u^{\top} \Sigma u\right]\right\}$ and $\psi_{X_{t}^{\mathrm{d}}}(u)=\exp \left\{t \int_{\mathbb{R}^{d} \backslash\{0\}}\left(\exp \left\{\mathrm{i} u^{\top} x\right\}-1-\mathrm{i} u^{\top} x 1_{\{\|x\|<1\}}\right) \nu(d x)\right\}$. Thus, a Lévy process is a continuous-time Markov process (satisfying 1-3) which recognizes a diffusion part, represented by the parameters $\gamma$ (known as the drift parameter) and $\Sigma$ (known as the diffusion coefficient), and a jump part, represented mainly through the Lévy measure $\nu$. Next, we will give two well-known examples of Lévy processes and list the types of jumps that can emerge depending on the behavior of $\nu$.

Example Let $\left\{N_{t}\right\}_{t \in \mathbb{R}_{+}}$be a Poisson process of rate $\lambda>0$, and consider a sequence of independent and identically distributed (IID) random variables (RVs) $\left\{Y_{j}\right\}_{j \in \mathbb{N}}$, of law $F$, independent of the Poisson process $\left\{N_{t}\right\}_{t \in \mathbb{R}_{+}}$. Assume that $\int_{\{|y|<1\}} y F(d y)=0$. Let $\left\{X_{t}\right\}_{t \in \mathbb{R}_{+}}$be the process defined for all $t \in \mathbb{R}_{+}$ as $X_{t}=\sum_{j=0}^{N_{t}} Y_{j}$, with $Y_{0}=0$. Thus, $\left\{X_{t}\right\}_{t \in \mathbb{R}_{+}}$is a Lévy process known as compound Poisson process whose CF can be written like (2) by taking $\gamma, \sigma^{2}=0$ and $\nu(d y)=\lambda F(d y)$ when we restrict to the case $d=1, \Sigma$ is equal to $\sigma^{2}$. That is,

$$
X_{t}(u)=E\left(\exp \left\{\mathbf{i} u X_{t}\right\}\right)=\exp \left\{t \lambda \int_{\mathbb{R}^{d}}(\exp \{\mathbf{i} u y\}-1) F(d y)\right\},
$$

$u \in \mathbb{R}$. The standard real Brownian motion is obtained by taking in $(2) \gamma=0, \sigma=1$ and $\nu \equiv 0$; when $\sigma>0$ and $\gamma \neq 0$ we say that we have a Brownian motion with drift $\gamma$ and diffusion coefficient $\sigma$, which is represented by $\gamma t+\sigma W_{t}$.

Path characteristics of a particular Lévy process can be known through the characteristics of its corresponding Lévy measure:

- If $\nu \equiv 0$, then the process has continuous paths.

- If $\int_{\mathbb{R}^{d} \backslash\{0\}} \nu(d x)<\infty$, then the process has jumps of finite activity, that is, it has finite jumps over any compact time horizon. When $\int_{\mathbb{R}^{d} \backslash\{0\}} \nu(d x)>0, \gamma=0$ and $\Sigma=0$, such processes can be modeled by a compound Poisson process.

- If $\int_{\mathbb{R}^{d} \backslash\{0\}} \nu(d x)=\infty$, then the process has jumps of infinite activity, that is, it has infinite jumps over any compact time horizon. Paths of these kind of processes are purely discontinuous.

For more details dedicated to an extensive treatment of Lévy processes see [6].

We are going to consider in this work that a Lévy flight is a pure-jump Lévy process, where between jumps we figure that the species is feeding (or reproducing) within some little or local neighborhood of the last stopped geographical point. As Lévy flights are characterized by its large jumps (due to its heavy-tailed jump distribution) it seems unrealistic to think that such jumps have infinite activity. Hence, 
we will assume that its jumps have finite activity, implying that a Lévy flight is actually modeled by a compound Poisson process.

\section{Simulation Scheme of Search Patterns}

First, we are going to review an Euler scheme for a general representation, where Lévy processes can be recognized. Later, we will exemplify it with an "intermediary" case.

\subsection{Euler Scheme for an Ornstein-Uhlenbeck Type Process}

Let $\left\{X_{t}\right\}_{t \in \mathbb{R}_{+}}$be a $\mathbb{R}^{d}$-valued Lévy process. We say that $\left\{Z_{t}\right\}_{t \in \mathbb{R}_{+}}$follows a Ornstein-Uhlenbeck type process if it satisfies the stochastic differential equation

$$
\left\{\begin{array}{c}
d Z_{t}=-\alpha Z_{t} d t+d X_{t} \\
Z_{0}=z_{0}
\end{array}\right.
$$

It can be noted that (4) has a unique strong solution, given by:

$$
Z_{t}=z_{0} \exp \{-\alpha t\}+\int_{0}^{t} \exp \{-\alpha(t-s)\} d X_{s}
$$

The Markovian property of such processes implies that for $0 \leq s \leq t$,

$$
Z_{t}=Z_{s} \exp \{-\alpha(t-s)\}+\exp \{-\alpha t\} \int_{s}^{t} \exp \{\alpha r\} d X_{r} .
$$

Hence, from this fact, we can use the following Euler scheme in order to approximate the process $\left\{Z_{t}\right\}_{t \in \mathbb{R}_{+}}$ [7]: for a fixed time horizon $T$, divide the interval $[0, T]$ as $0=t_{0}<t_{1}<\ldots<t_{n}=T$, with $t_{j}-t_{j-1}=\delta$ for all $j=1, \ldots, n$. Therefore, approximation of (5)-(6) is achieved by the following recursive scheme:

$$
Z_{t_{j}}=\exp \{-\alpha \delta\}\left(Z_{t_{j-1}}+X_{t_{j-1}}^{\star}\right)
$$

where $X_{t_{j-1}}^{\star}=\int_{t_{j-1}}^{t_{j}} \exp \{\alpha s\} d X_{s} \stackrel{\mathcal{L}}{=} \int_{0}^{\delta} \exp \{\alpha s\} d X_{s} \approx \sum_{j=1}^{\lceil\delta / h\rceil} \exp \{\alpha j h\} X_{h}$, with $0 \leq h \leq \delta$ small enough, and where $\lceil\cdot\rceil$ is the ceiling function.

Under simple algebraic manipulations, it can be shown that this kind of schemes are equivalent to the ones studied in [8], and then, its convergence rate is proportional to $(n / \log (n))^{1 / \rho}$, where $\rho>0$ is a parameter that depends on the behavior of the Lévy measure associated to $\left\{X_{t}\right\}_{t \in \mathbb{R}_{+}}$in a neighborhood of the origin; in other words, this parameter depends on the jump activity within a neighborhood of zero. We strongly recommend [8] for a detailed discussion about this dependency.

\subsection{An "Intermediary" Case}

Here, we will briefly look at an "intermediary" situation of a jump Lévy process, i.e. neither a Brownian pattern nor a Lévy flight one, where the jump doesn't fellow a heavy-tailed distribution. Consider $X_{t}=\sum_{j=0}^{N_{t}} \mathcal{Y}_{j}$, such that:

$$
\mathcal{Y}_{j} \sim \mathcal{N}_{2}(\mathcal{U}, \Sigma)
$$

where " $\mathcal{N}_{2}(\mathcal{U}, \Sigma)$ " means "bivariate normal distribution with vector mean $\mathcal{U}$ and covariance matrix $\Sigma$ ". It is easy to check that such jumps do not satisfy (1).

In certain sense, the above process is closer to the Brownian motion patterns than the Lévy flight one. Indeed, for the re-scaled space-time transformation $\mathcal{M}_{t}^{n}=\left(X_{n t}-E\left(X_{n t}\right)\right) / \sqrt{n}=\left(X_{n t}-n \lambda t \mathcal{U}\right) / \sqrt{n}$, we have that, for all $t>0, \mathcal{M}_{t}^{n}$ converges in law towards a zero-mean normal random variable whose variance 


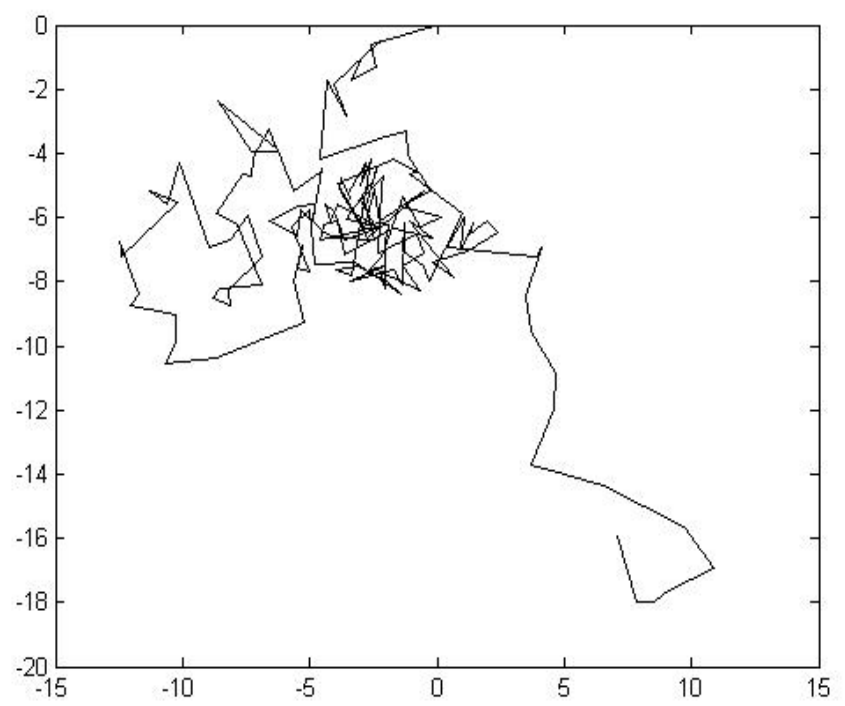

Figure 3. A compound Poisson process with rate $\lambda=1 / 3$ (mean time between jumps $\approx 2,92$ ), whose jumps follow a bivariate normal random distribution with mean $\mathcal{U}=0$ covariance matrix $\Sigma=1000 I_{2}$, where $I_{2}$ is the $2 \times 2$ identity matrix.

is proportional to $t$, whenever $n \rightarrow \infty$, by the Central Limit Theorem. Moreover, from a functional perspective, $\left\{\mathcal{M}^{n}\right\}_{n \in \mathbb{N}}$ is a sequence of elements in $D\left(\Omega, \mathbb{R}^{2}\right)$, which converges functionally towards a bivariate Brownian motion, which is an element of the set of continuous functions $C\left(\Omega, \mathbb{R}^{2}\right)$ (see $\left.[9]\right)$. Roughly speaking, this compound Poisson process is conditionally a random walk. Nevertheless, if we consider a Lévy flight, such a re-scaled space-time transformation could not lead to a Brownian pattern, because the heavy-tailed property of the jump law often yields the non existence of any of its moments (for example, the jumps of the process pictured in Figure 2 follow a Cauchy distribution, which has no moments). What does this mean in practice? It says that if we register such an "intermediary" process, the observed pattern will start to look like a Brownian motion like Figure 1 when "zooming out" the observational area. Therefore, we have to keep in mind at which scale we are going to start analyzing this kind of phenomena.

Likelihood function of a compound Poisson process Our Lévy process can be written as $X_{t}=$ $\sum_{j=0}^{N_{t}} \mathcal{Y}_{j}$, where $\left\{N_{t}\right\}_{t \in \mathbb{R}_{+}}$is a Poisson process with rate $\lambda>0$, independent of the IID sequence $\left\{\mathcal{Y}_{j}\right\}_{j \in \mathbb{N}}$, which takes value $\mathbb{R}^{d}$. If we assume that the law of the $\mathcal{Y}_{j}$ 's can be written as $F^{\theta}(d y)=f^{\theta}(y) d y$, that is, the jumps have a probability density function $f^{\theta}$, where $\theta$ denotes the parameters involved, we can construct the likelihood function in order to further estimate $\theta$.

Let $A$ be any measurable set of $\mathbb{R}^{d}$. Then, for all $t \in \mathbb{R}_{+}$we have,

$$
P\left(X_{t} \in A\right)=\sum_{n} P\left(X_{t} \in A \mid N_{t}=n\right) P\left(N_{t}=n\right),
$$

by the "law of total probability". Then, if we observe the $n$ jumps (the lengths of the species displacement registered through of adequate coordinates on the plane $\mathbb{R}^{2}$, for example) during the time interval $[0, t]$, (8) can be written as:

$$
\begin{gathered}
P\left(X_{t} \in A\right)=\int \cdots \int_{R} f^{\theta}\left(y_{1}, \ldots, y_{n}\right) d y_{1} \cdots d y_{n} \times \exp \{-\lambda t\} \frac{(\lambda t)^{n}}{n !} \\
=\int \cdots \int_{R} \prod_{i=1}^{n} f^{\theta}\left(y_{i}\right) d y_{1} \cdots d y_{n} \times \exp \{-\lambda t\} \frac{(\lambda t)^{n}}{n !}
\end{gathered}
$$


where $R$ is a suitable region in $\mathbb{R}^{n d}$. Thus, from (9) we obtain the likelihood function $L(\theta, \lambda)$ :

$$
L(\theta, \lambda)=\lambda^{n} \exp \{-\lambda t\} \prod_{i=1}^{n} f^{\theta}\left(y_{i}\right)=\exp \{-\lambda t\} \prod_{i=1}^{n} m^{\theta}\left(y_{i}\right),
$$

where $\lambda f^{\theta}(y) d y=m^{\theta}(y) d y=\nu^{\theta}(d y)$, with $\nu^{\theta}$ the corresponding Lévy measure. From (10), the maximum likelihood estimator of $\lambda, \widehat{\lambda}$, is given by:

$$
\widehat{\lambda}=\left(\frac{1}{n} \sum_{i=1}^{n} \tau_{i}\right)^{-1}
$$

where the $\tau_{i}$ 's are the times between the $n$ jumps.

\section{Discussion}

From the "intermediary" pattern of displacement pictured in Figure 3, an important question arises: How can we known if there is abundance or not of food? Such an intermediary pattern described by the movement of a certain species may indicate the beginning of a change in its food distribution. The two opposite situations (abundance and lack of food) can be suitably pictured by a Brownian motion and a Lévy flight, respectively. These patterns are of different nature: the first one has continuous paths and the second one is a pure-jump process. When we account for displacement data, a first step would be to recognize the nature of the displacement; that is, by assuming that the process belongs to the family of Lévy processes, we would contrast statistically whether the process has jumps or not [10]. If the process has jumps, we would proceed by studying if such jumps come from a heavy-tailed distribution. In general, this procedure can be difficult to analyze. Nevertheless, some subclasses of heavy-tailed distributions have been analyzed, as the power law distributions (see, e.g., [11], [12], [13] and [14]), but which do not capture all the heavy-tailed distributions. In fact, the existence of a density function behaving like $x^{-a}$ in its tails is assumed for these cases. Thus, according to our definition (1), this general analysis for all the heavy-tailed distributions remains as an open problem. Precisely, if $\mathcal{X}$ is "jump displacement length" (which can be assumed to be absolutely continuous), then it has a heavy-tailed distribution if and only if,

$$
\lim _{x \uparrow \infty} \frac{\mathrm{d} \ln \left(\varphi_{\mathcal{X}}(x)\right)}{\mathrm{d} x}=\lim _{x \uparrow \infty} f_{\mathcal{X}}(x) \varphi_{\mathcal{X}}(x)=0,
$$

where $\varphi_{\mathcal{X}}(\cdot)=[P(\mathcal{X}>\cdot)]^{-1}$ and $f_{\mathcal{X}}(\cdot)$ is the probability density of $\mathcal{X}$. Thus, a statistical test for this fact remains to be implemented.

Acknowledgements This work was supported by the FONDECYT 3140613 project.

\section{References}

1. N. Humphries, N. Queiroz, J. Dyer, N. Pade, M. Musy, K. Schaefer, D. Fuller, J. Brunnschweiler, T. Doyle, J. Houghton, G. Hays, C. Jones, L. Noble, V. Wearmouth, E. Southall, and D. Sims, "Environmental context explains lévy and brownian movement patterns of marine predators," Nature, vol. 465, pp. 1066-1069, 2010.

2. S. Petrovskiia, A. Mashanovab, and V. Jansenb, "Variation in individual walking behavior creates the impression of a lévy flight," PNAS, vol. 108, no. 21, pp. 8704-8707, 2011.

3. A. Reynolds and C. Rhodes, "The lévy flight paradigm: random search patterns and mechanisms," Ecology, vol. 90 , no. 4 , pp. 877-887, 2009.

4. G. Viswanathan, "Fish in lévy-flight foraging," Nature, vol. 465, pp. 1018-1019, 2010.

5. G. Viswanathan, V. Afanasyev, S. Buldyrev, E. Murphy, P. Prince, and H. Stanley, "Lévy flight search patterns of wandering albatrosses," Nature, vol. 381, pp. 413-415, 1996.

6. K. Sato, Lévy Processes and Infinitely Divisible Distributions. Cambridge university press, 1999.

7. L. Valdivieso, "Likelihood inference in processes of ornstein-uhlenbeck type," Ph.D. dissertation, Katholieke Universiteit Leuven, Faculteit Wetenschappen, 2007. 
8. J. Jacod, "The euler scheme for lévy driven stochastic differential equations: limit theorems," The Annals of Probability, vol. 32, no. 3A, pp. 1830-1872, 2004.

9. R. Darling. (2002) Fluid limits of pure jump markov processes: a practical guide. [Online]. Available: http://arxiv.org/abs/math/0210109

10. Y. Aït-Sahalia and J. Jacod, "Testing for jumps in a discretely observed process," The Annals of Statistics, vol. 37, no. 1, pp. 184-222, 2009.

11. B. S. Raposo, E and, M. da Luz, H. Stanley, and G. Viswanathan, "Dynamical robustness of lévy search strategies," Physical Review Letters, vol. 91, no. 24, pp. 240 601-1-240 601-4, 2003.

12. B. S. Raposo, E and, M. da Luz, M. Santos, H. Stanley, and G. Viswanathan, "LÃlvy flights and random searches," Journal of Physics A: Mathematical and Theoretical, vol. 42, pp. 434003-1-434003-23, 2009.

13. G. Viswanathan, S. Buldyrev, S. Havlin, M. da Luz, E. Raposo, and H. Stanley, "Lévy flight search patterns of wandering albatrosses," Nature, vol. 401, pp. 911-914, 1999.

14. G. Viswanathan, E. Raposo, and M. da Luz, "Lévy flights and superdiffusion in the context of biological encounters and random searches," Physics of Life Reviews, vol. 5, pp. 133-150, 2008. 